\title{
In Vivo Synthesis of EuSe Nanoparticles and its Cytotoxicity against Cancer Cells
}

\author{
Ji Min Seo, Eun Bee Kim, Tae Jung Park \\ Department of Chemistry, Chung-Ang University \\ 84 Heukseok-ro, Dongjak-gu, Seoul 06974, Republic of Korea \\ s6954677@gmail.com; sivan8600@naver.com; tjpark@cau.ac.kr
}

\begin{abstract}
Nanotechnology strives to combine new materials for development of nanoparticles (NPs). As NPs exhibit unique optical, electronic, and magnetic properties depending on their composition, developing safe, cost-effective and environmentally friendly technologies for synthesis of NPs have become an important issue. In the present study, in vivo synthesis of europium selenide (EuSe) NPs was performed using recombinant Escherichia coli cells expressing heavy-metal binding proteins (HMBPs), phytochelatin synthase and metallothionein. The formation of EuSe NPs was verified by characterization using UV-vis spectrophotometry, spectrofluorometry, $\mathrm{X}$-ray diffraction, energy dispersive X-ray and transmission electron microscopy. The synthesized EuSe NPs exhibited high fluorescence intensity with strong magnetic properties. Furthermore, anticancer effect of EuSe NPs against cancer cell line was investigated. This result showed that the synthesized EuSe NPs have a great potential as bioimaging tools and drug carrying agents in biomedical fields.
\end{abstract}

Keywords: Heavy-metal binding protein, In vivo synthesis, EuSe nanoparticles, Anti-cancer effect

\section{Introduction}

In recent years, various methods have been developed in order to synthesize nanoparticles (NPs) with specific properties while striving to consume less energy, increase efficiency, and avoid the production of toxic byproducts [1]. From this, the global tendency to employ eco-friendly processes [2] has led to a growing interest in developing methods using biocatalysts such as bacteria [3] fungi, actinomycete [4], yeast, and plant cells, which possess unique biocatalysis properties. Hence, it has been important to develop synthetic strategies that are simple, cost-effective and environment friendly. However, most of reported biological synthesis mainly focused on the formation of gold (Au), silver (Ag), silica ( $\mathrm{Si}$ ), and iron oxide $\left(\mathrm{Fe}_{3} \mathrm{O}_{4}\right)$ NPs [5] that were already synthesized and evaluated by many researchers using chemical means. Its synthetic strategies and significance in biomedical and electrochemical fields has been well defined over the past 20 years and is not novel to us anymore. Thus, development of novel NPs with unique optical, electronic, and magnetic properties has triggered a new interest among researchers in nanotechnology [6].

In this paper, we report in vivo synthesis of europium selenide (EuSe) NPs by using recombinant Escherichia coli expressing Arabidopsis thaliana phytochelatin synthase (AtPCS) and Pseudomonas putida metallothionein (PpMT). Phytochelatin (PC) and metallothionein (MT) are cysteine-rich, heavy-metal binding proteins. PCs are enzymatically synthesized peptides and MTs are gene-encoded polypeptides. They have demonstrated high metal-binding affinity and accumulation capacity inside cells. We genetically designed and engineered E. coli to express a PC synthase (PCS) of $A$. thaliana along with an MT of $P$. putida for determining the synergistic effect of the PpMT-AtPCS fusion protein on metal component assembly for NP synthesis [7-10]. The formation of EuSe NPs was first observed through color change of the reaction mixture from yellowish-white to orange-pink and the characterization was carried out to show its unique properties such as high fluorescence intensity and strong magnetic fields. Synthesized EuSe NPs not only exhibited its ability as bioimaging tools but anticancer effect against cancer cells proving its potential as drug carrying agents as well.

\section{Experimental Section}

\subsection{Preparation of EuSe NPs}

Recombinant E. coli BL21(DE3) co-expressing AtPCS and PpMT was prepared according to the methods described in a previous work [9]. Briefly, recombinant E. coli cells expressing pTJ1-PpMT-AtPCS were grown at $37^{\circ} \mathrm{C}$ for $2 \mathrm{~h}$ in 100 
$\mathrm{mL}$ of Luria-Bertani (LB) medium. When the absorbance value of at $600 \mathrm{~nm}\left(\mathrm{O}_{\mathrm{D}} \mathrm{D}_{600}\right)$ reached $0.4,0.5 \mathrm{mM}$ isopropyl- $\beta$-Dthiogalactopyranoside (IPTG) was added for induction of PCS and MT. EuSe NPs were produced after incubation of another $1 \mathrm{~h}$ followed by slow injection of Eu and Se precursors at final concentration of 1, 2, 3, 4, $5 \mathrm{mM}$. 0.5 M stock solution of precursors used were prepared using $\mathrm{EuCl}_{3} \cdot 6 \mathrm{H}_{2} \mathrm{O}, \mathrm{SeO}_{2}$ (Sigma-Aldrich, St. Louis, MO), and deionized (DI) water (Direct$\mathrm{Q}^{\circledR} 3$ Water Purification System, Millipore, Billerica, MA). The samples mixed with europium and selenium precursors were further incubated for $4 \mathrm{~h}$ at $30^{\circ} \mathrm{C}$ in a shaking incubator (SI-600R, Jeio-Tech, Korea). After constant shaking, color change of the solution was observed from yellowish-white to orange-pink proving the formation of EuSe NPs. The synthesized NPs with recombinant $E$. coli BL21(DE3) cells were centrifuged at 3,500 rpm $(2,691 \times \mathrm{g})$ for $15 \mathrm{~min}$ and its supernatant was removed. The leftover pellet was washed twice with phosphate-buffered saline (PBS, pH 7.4, Sigma-Aldrich) and ultrasonicated until the solution became clear. The formed EuSe NPs were collected by centrifugation and washed with ethanol and DI water several times. Synthesized EuSe NPs solution was stored at $4{ }^{\circ} \mathrm{C}$ and sonicated before use.

\subsection{Characterization of EuSe NPs}

UV-visible absorption spectroscopy was used to monitor the plasmon absorption of the $3 \mathrm{mM}$ EuSe NPs and compared with PpMT-AtPCS without NPs using a Jasco V-670 spectrometer with $1 \mathrm{~nm}$ spectral resolution. Fluorescence spectra of the cell suspension with 1, 2, 3, 4, and $5 \mathrm{mM}$. of EuSe NPs were analyzed using a multi-mode microplate reader (BioTek Synergy H1, Winooski, VT) with excitation at $320 \mathrm{~nm}$. X-ray diffraction (XRD) measurement was carried out in a Rigaku X-ray diffractometer (New D8-Advance/Bruker-AXS, Berlin, Germany) with $\mathrm{Cu}-\mathrm{K} \alpha$ X-ray source $(\lambda=1.54056 \AA)$ at a generator voltage $40 \mathrm{kV}$. The high-resolution (HR) structure analysis of NPs was performed by TEM (Tecnai G2 F30 STWIN; FEI, Hillsboro, OR) at an accelerating voltage of $300 \mathrm{kV}$ with $0.20 \mathrm{~nm}$ point resolution. The samples for TEM analysis were prepared by dispersing the NPs in 1 M PBS solution and placing a droplet on the copper grid with 300 grid mesh with $3 \mathrm{~nm}$ diameter. The excess solvent was evaporated at room temperature. The elemental analysis of NPs was carried out by the energy dispersive X-ray (EDX) analyzer associated with TEM, at an accelerating voltage of $20 \mathrm{kV}$. Finally, magnetic property of the NPs was simply shown by separating NPs from the solution using neodium magnet.

\subsection{Cytotoxicity assay of EuSe NPs}

HeLa and 293T cells were grown in Dulbecco's Modified Eagle's Medium (DMEM) supplemented with 10\% (v/v) fetal bovine serum and non-essential amino acids at $37^{\circ} \mathrm{C}$ in humidified conditions maintained by passing $5 \%(\mathrm{w} / \mathrm{v}) \mathrm{CO}_{2}$. Effect of EuSe NPs on the viability of HeLa cells was determined by the conventional 3-(4, 5-dimethylthiazolyl-2)-2, 5diphenyltetrazolium bromide (MTT) reduction assay [11]. Therefore, $5 \times 10^{4}$ cells/well were cultured in a 24-well plate. After $24 \mathrm{~h}$, the culture medium was removed and fresh medium containing $1.0-2.0 \times 10^{3} \mu \mathrm{M}$ EuSe NPs were added to each well and placed in the incubator for additional $24 \mathrm{~h}$. Cells without the NPs were used as positive control. After $24 \mathrm{~h}$ incubation, medium was removed and $300 \mu \mathrm{L}$ of MTT solution $(1 \mathrm{mg} / \mathrm{mL}$ in PBS) was added to each well. The plate was incubated for $4 \mathrm{~h}$ at $37^{\circ} \mathrm{C}$ in a $\mathrm{CO}_{2}$ incubator and then $600 \mu \mathrm{L}$ of medium was replaced in each well with equal volume of DMSO-methanol mixture (50:50). The resultant was mixed thoroughly on a plate shaker. After dissolution of crystals, absorbance was measured at $570 \mathrm{~nm}$ with a multi-mode microplate reader. Concentration $(200 \mu \mathrm{M})$ lower than $\mathrm{IC}_{50}(300$ $\mu \mathrm{M})$ was used for studying toxicity of EuSe NPs including cell viability (MTT assay) and ROS levels in HeLa cells.

To observe cell morphology, HeLa and 293T cells $\left(1 \times 10^{4}\right.$ cells $\left./ \mathrm{mL}\right)$ were grown in DMEM (with $10 \%(\mathrm{v} / \mathrm{v})$ fetal bovine serum and non-essential amino acids) at $37^{\circ} \mathrm{C}$ in humidified conditions maintained by passing $5 \%\left(\mathrm{w} / \mathrm{v}\right.$ ) $\mathrm{CO}_{2}$ and treated $200 \mu \mathrm{M}$ EuSe NPs. After incubation for 12 and $24 \mathrm{~h}$, the cells were observed under a bright-field microscope (Jenoptik, Germany). EuSe-treated $(2.5 \mu \mathrm{g} / \mathrm{mL})$ cells were observed and compared with untreated cells as a negative control.

\section{Results and Discussion}

\subsection{Characterization of EuSe NPs}

Fig. 1a shows the UV-vis absorption spectra of the synthesized EuSe NPs and PpMT-AtPCS. The optical spectra show the presence of EuSe NPs with plasmonic band at $550 \mathrm{~nm}$. This specific absorption band was not observed in PpMT-AtPCS revealing the formation of EuSe NPs. Fig. 2b shows increase in fluorescent intensity of EuSe NPs with increase in concentrations of $1,2,3,4$, and $5 \mathrm{mM}$, respectively. 
a

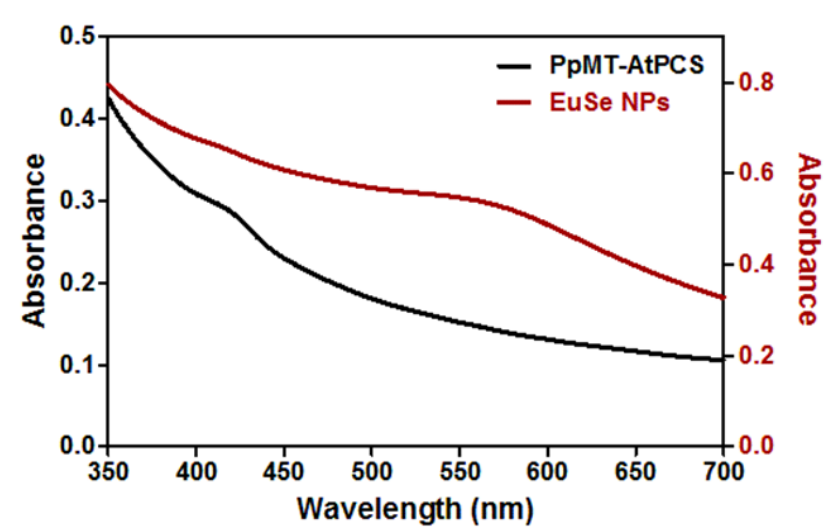

b

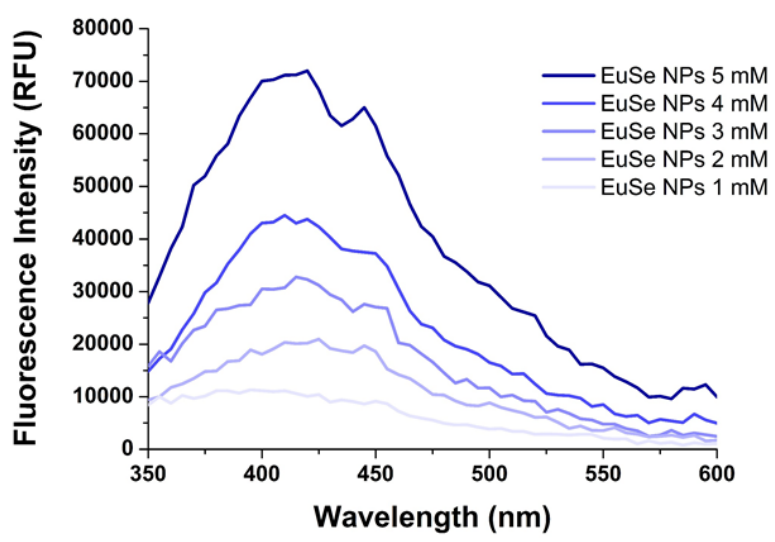

Fig. 1: (a) UV-vis absorption spectra of $3 \mathrm{mM}$ EuSe NPs and PpMT-AtPCS without metal ions. (b) Fluorescence spectra of EuSe NPs with different concentrations in recombinant $E$. coli cells.

The size and morphology of EuSe NPs synthesized in the recombinant E. coli were analyzed by TEM. Fig. 2a shows the EuSe NPs synthesized by recombinant cells. Black dots around cells are EuSe NPs formed by interacting with recombinant proteins (Fig. 2b). After cell lysis, presence of NPs with a size of about $3 \mathrm{~nm}$ has been observed in aqueous solution (Fig. 2c). Electron diffraction analysis shows diffuse ring patterns and crystal spots with a d-spacing of $3.0 \pm 0.03$ $\AA$, which indicates a randomly oriented fine crystalline nanostructure (Fig. 2d).

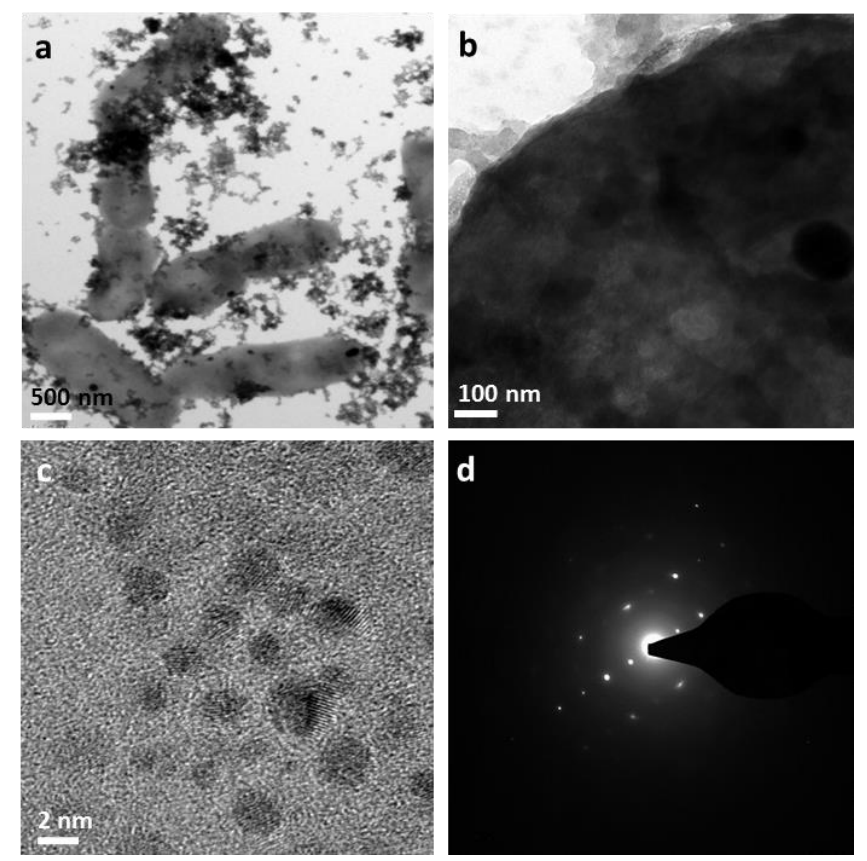

Fig. 2: TEM images of: (a and b) EuSe NPs with recombinant $E$. coli cells in different magnifications, (c) EuSe NPs in aqueous solution after cell lysis, (d) electron diffraction pattern of multi-layer region of EuSe.

The XRD peaks obtained in Fig.3 were assigned using the standard data from JCPDS card (File No. 00-001-0853) to the cubic (101) EuSe phase. The XRD pattern showed peaks at $2 \theta$ values of $23.508^{\circ}, 29.699^{\circ}$, and $43.650^{\circ}$ corresponding to the $h k l$ planes (100), (101), and (012), respectively. Assuming a homogeneous strain across the EuSe films, the average particle size $(d)$ was estimated from the full-width at half-maximum (FWHM) of the (101) peak by using the Debye Scherrer equation, $\mathrm{d}=0.9 \lambda / \mathrm{B} \cos \theta$, where $\lambda(1.5418 \AA)$ is the wavelength of $\mathrm{X}$-rays $(\mathrm{Cu} \mathrm{K \alpha}), \theta$ is the angle of reflection, and $\mathrm{B}$ is the widening of the Bragg XRD peak [12]. 


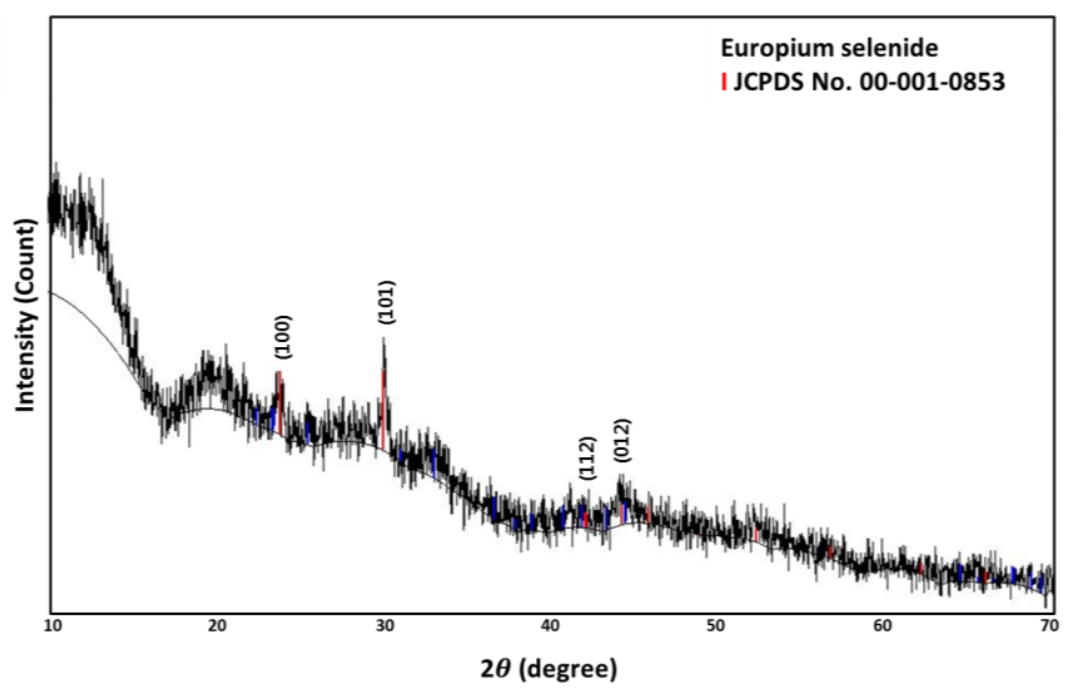

Fig. 3: XRD pattern of synthesized EuSe NPs.

Fig. 4 illustrates an EDX spectrum of the EuSe NPs. The spectrum confirms the presence of europium, selenium in the synthesized NPs proving a successful formation of EuSe NPs. The magnetic property of EuSe NPs is shown in Fig. 5. 3 $\mathrm{mM}$ EuSe NPs have been attracted to neodium magnet within 10 min showing its strong magnetic behavior.

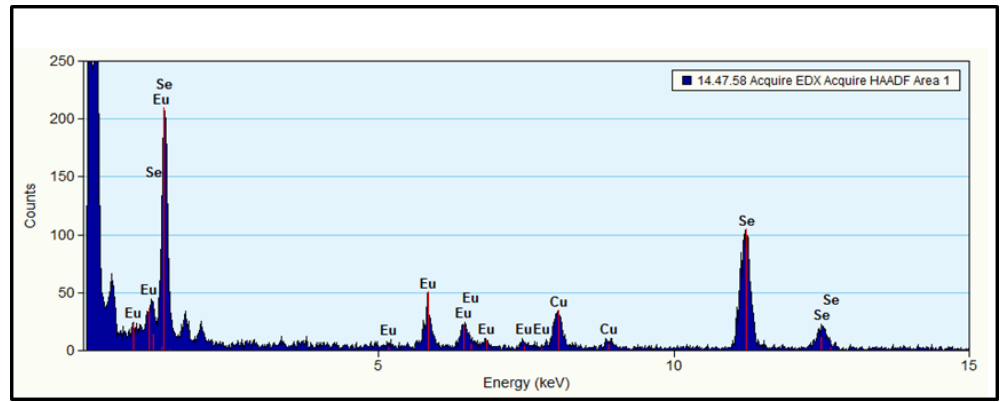

Fig. 4: Energy dispersive X-ray (EDX) analysis of EuSe NPs.

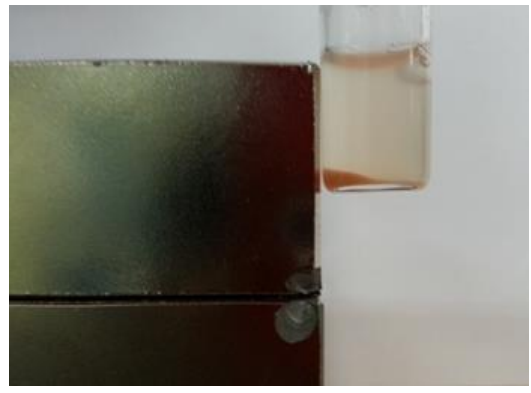

Fig. 5: EuSe NPs with a neodium magnet $50 \mathrm{~mm} \times 50 \mathrm{~mm} \times 50 \mathrm{~mm}$ (DongHwa magnet, Korea) at room temperature for $10 \mathrm{~min}$.

\subsection{Effect of EuSe NPs on viability and morphology in 293T and HeLa cells}

Herein, we evaluated the cytotoxicity of synthesized EuSe NPs by measuring the viability of 293T and HeLa cells. Fig. 6 shows that cell viability is highly dependent on concentration of EuSe NPs. The $\mathrm{IC}_{50}$ value was found to be $300 \mu \mathrm{M}$. Concentration between 100 and $400 \mu \mathrm{M}$ showed higher cell viability in 293T cell compared to HeLa cell. Thus, concentration $(200 \mu \mathrm{M})$ lower than $\mathrm{IC}_{50}$ was used for studying the toxic effects of EuSe NPs on HeLa cell, and it was observed that the cell viability decreased with increased incubation time (Fig. 7). Typical morphology of HeLa cells was not observed in EuSe NPtreated cells but wrinkled cells with shrunken nuclei were found. This reveals anticancer properties of EuSe NP as small NPs have large surface area per unit mass making NPs very reactive in the cellular environment [13]. 

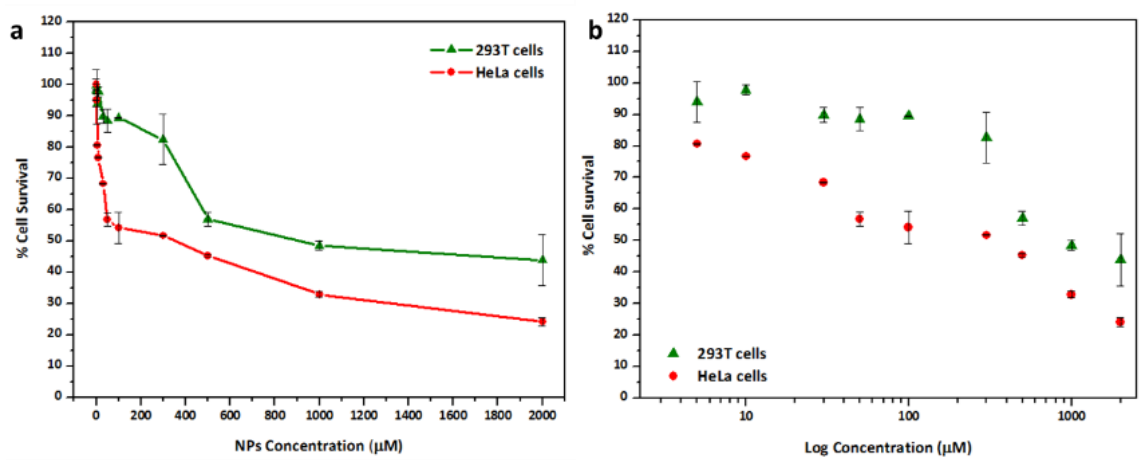

Fig. 6: Cytotoxic effect of EuSe NPs on $293 \mathrm{~T}$ and HeLa cell lines (a). Graph changed to log scale (b).

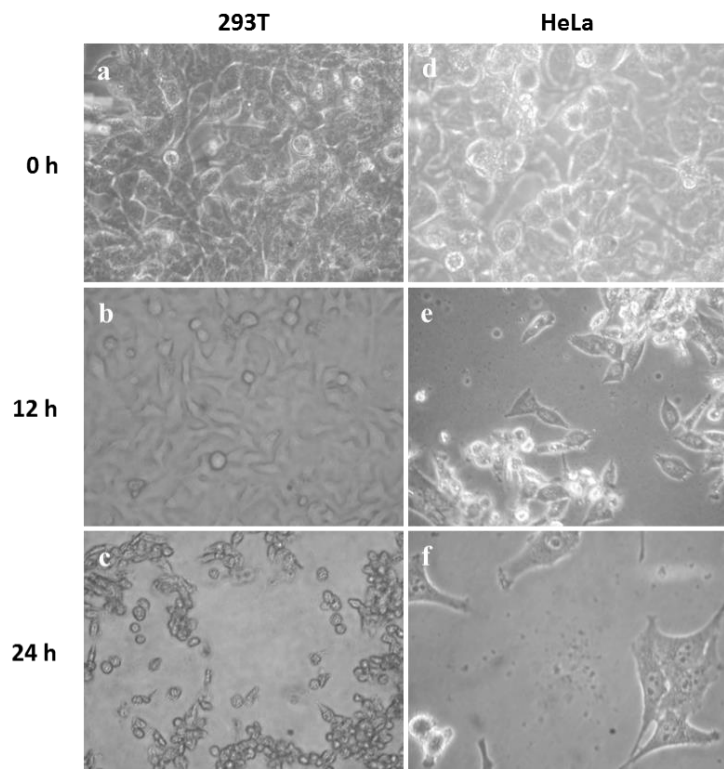

Fig. 7: Effect of EuSe NPs on 293T (a-c) and HeLa cell (d-f) morphology with incubation time of 0,12 and 24 h.

\section{Conclusion}

In this work, a successful synthesis of EuSe NPs using PpMT-AtPCS has been described. The synthesized EuSe NPs were thoroughly characterized to prove its morphology, optical and magnetic properties. UV-visible analysis showed absorption band of EuSe NPs at $550 \mathrm{~nm}$ and XRD analysis confirmed its crystalline formation. TEM images also confirmed the formation of EuSe NPs through the recombinant proteins, HMBPs. In vitro studies performed on HeLa and 293T cells showed its cytotoxic effects against cancer cell line. This indicates EuSe NPs can be utilized as promising drug carrying agents and cell imaging materials with anticancer effects in targeted drug delivery against cancer. The recombinant $E$. coli system expressing heavy-metal binding proteins, PpMT-AtPCS, can also be applied in synthesizing of various types of metal NPs with desired optical, electronic, chemical, and magnetic properties.

\section{Acknowledgements}

This work was supported by a grant from the Korean Health Technology R\&D Project, Ministry of Health \& Welfare, Republic of Korea (HI16C1553), and by Advanced Production Technology Development Program, Ministry of Agriculture, Food and Rural Affairs (315050-2).

\section{References}

[1] A. Panáček, et al., "Silver colloid nanoparticles: synthesis, characterization, and their antibacterial activity," J. Phys. Chem. B., vol. 110, no. 33, pp. 16248-26253, 2006. 
[2] D. Mandal, M. Bolander, D. Mukhopadhyay, G. Sarkar, and P. Mukherjee, "The use of microorganisms for the formation of metal nanoparticles and their application," Appl. Microbiol. Biotechnol., vol. 69, no. 5, pp. 485-492, 2006.

[3] P. Mohanpuria, N. Rana, and S. Yadav, "Biosynthesis of nanoparticles: technological concepts and future applications." J. Nanopart., vol. 10, no. 3, pp. 507-517, 2008.

[4] S. Basavaraja, et al., "Extracellular biosynthesis of silver nanoparticles using the fungus Fusarium semitectum," Mater. Res. Bull., vol. 43, no. 5, pp. 1164-1170, 2008.

[5] K. Narayanan and N. Sakthivel, "Biological synthesis of metal nanoparticles by microbes," Adv. Colloid. Interface Sci., vol. 156, no. 1, pp. 1-13, 2010.

[6] C. Medina, et al., "Nanoparticles: pharmacological and toxicological significance," Br. J. Pharamacol., vol. 150, no. 5, pp. 552-558, 2007.

[7] J. H. Jung, et al., "Homogeneous biogenic paramagnetic nanoparticle synthesis based on a microfluidic droplet generator," Angew. Chem. Int. Ed., vol. 124, no. 23, pp. 5732-5735, 2012.

[8] K. G. Lee, et al., "In vitro biosynthesis of metal nanoparticles in microdroplets," ACS Nano., vol. 6, no. 8, pp. 69987008, 2012.

[9] T. J. Park, et al., "In vivo synthesis of diverse metal nanoparticles by recombinant Escherichia coli," Angew. Chem. Int. Ed., vol. 122, no. 39, pp. 7173-7178, 2010.

[10] J. M. Seo, et al., "Self-assembly of biogenic gold nanoparticles and their use to enhance drug delivery into cells," Colloids Surf. B., vol. 135, pp. 27-34, 2015.

[11] Z. Hu, et al., "Photodynamic anticancer activities of water-soluble $\mathrm{C}_{60}$ derivatives and their biological consequences in a HeLa cell line," Chem. Biol. Interact., vol. 194, no. 1, pp. 86-94, 2012.

[12] B. Cullity, Elements of X-ray Diffraction. MA: Addison-Wesley Pub. Co., 1956.

[13] K. Donaldson, et al., "Carbon nanotubes: a review of their properties in relation to pulmonary toxicology and workplace safety,” Toxicol. Sci., vol. 92, no. 1, pp. 5-22, 2006. 